\section{The sexiness of sex ratios}

\author{
Sex Ratios: Concepts and Research Methods \\ ICW Hardy \\ Cambridge University Press, Cambridge, UK. 2002; 424 pp. \\ £32.95. ISBN 0-521-66578-7
}

Heredity (2003) 91, 6-7. doi:10.1038/sj.hdy.6800273

Reviewed by DM Shuker

Sex ratios are sexy. That at least is the feeling you get from reading this book, in which many of the authors enthusiastically review the now comprehensive theoretical and empirical basis of sex ratio research. However, for all this enthusiasm, the most satisfying aspect of this book is undoubtedly the mature, self-critical nature of these reviews. Sex ratios may be sexy, but that is not to say we completely understand them.

The book, as the subtitle suggests, is very much about concepts and research methods, and as such provides a very clear set of methodological tools for those entering the sex ratio field (or indeed those already in it), in terms of developing theory, doing experiments, and, most importantly of all, analysing data. By doing so, many of the chapters provide insight, even if they are outside the reader's usual taxonomic remit, as my increasingly large collection of notes for this review testified.

The book is in six parts, commencing with sex ratio theory. The opening chapter by Seger and Stubblefield sets the standard by providing a clear account of the basic theory, put in a very reader-friendly historical context. Pen and Weissing then expand the theory base, outlining some of the more derived theoretical tools required for more complex modelling, considering, for example, sex ratios in organisms with more complicated life-histories. The second part of the book may well be the most useful, dealing with the statistical analysis of sex ratio data in a number of contexts. In particular, Wilson and Hardy's chapter should become the standard text for sex ratio statistics. They propose, and justify, that appropriate generalized linear models (GLMs) should become the statistical framework of choice where possible, and offer a lucid introduction into their use and benefits. Put simply, GLMs add power. We should all use them when we can.

Part 3 considers more mechanistic issues, looking at sex determination and the genetics of sex ratio. In terms of both vertebrate and invertebrate sex determination, our ignorance is the main take-home message, albeit a stimulating one. Stouthamer et al. then give a very good review of sex ratio distorters, which may prove to be unnervingly common in invertebrates, and a guide to techniques for their discovery and identification. As in many of the chapters, this aspect of sex ratio can support (and has supported) a volume in its own right, but the emphasis on practical issues makes the contribution valuable. Parts 4 and 5 contain chapters on empirical sex ratio research, with generous taxonomic breadth (wasps, mites, aphids, birds and mammals, and humans in Part 4, protozoa and plants in Part 5). All these chapters put empirical data in the context of theory, such as the familiar Trivers-Willard hypothesis or Hamilton's theory of local mate competition (LMC). Throughout it is well recognized that theory may be incomplete, or yet to be fully tested. Many of the authors are also only too aware that multiple selective forces may well influence sex allocation. This candour is refreshing and bodes well for the next generation of empirical work.

The final part returns to more theoretical matters, and perhaps should be read with Part 1 . I particularly enjoyed Kvarnemo and Ahnesjö's chapter on the operational sex ratio (OSR), as while much of the sex ratio field is concerned with sex ratios as an end point (in particular, if they are adaptive for whatever reason), sex ratios influence other population processes, such as sexual selection. This is most obvious for the generally nonadaptive (for the host) sex ratio effects of the sex ratio distorters described by Stouthamer et al. Changes in OSR and the resulting effects on sexual selection are a clear consequence of such sex ratio distorters. The final two chapters have different takes on the successes and failures of sex ratio research. Orzack gives a sober review of LMC theory, suggesting its occurrence has been vastly over-rated and the models poorly tested (if tested at all), with little attention given to alternatives. Fortunately, as I have already intimated, the authors of the empirical chapters seem to appreciate the need to consider multiple influences on sex ratio, even if one particular selective force, such as LMC, is likely to predominate. The limitations highlighted by Orzack are in some ways turned to everyone's advantage in the concluding chapter by West and Herre. While they stress more optimistically the success of sex ratio theory and experiment, they suggest that sex allocation should be used as a tool to see just how good our models are and why they fail. The relatively straightforward assumptions and clear predictions of the simplest models, and the fact that the sex ratio is so clearly related to fitness, means that very real progress can be made advancing theory and experiment together, slowly capturing more reality (for instance, dispelling some of the 'lies' concerning LMC Orzack suggests we have been left with). We can then start to ask more fundamental questions concerning adaptation in general, as well as turning to sex ratios to ask questions about things such as population ecology. West and Herre therefore offer a more positive outlook on the same issue of models and reality.

Who should read this book? Workers in or close to the field should find it enormously useful and 
thoughtful. For those outside the field there is also much to recommend it, although Sex Ratios does not perhaps provide an overall synthesis. Despite the clarity of the individual chapters, and the remarkably cohesive feel of what is after all an edited collection, this may well have to wait for a single authored volume.
DM Shuker

ICAPB, Ashworth Laboratories, University of Edinburgh King's Buildings, Edinburgh EH9 3JT, UK

E-mail: david.shuker@ed.ac.uk 